\title{
RESEARCH PAPER \\ INFLUENCE OF DRYING TEMPERATURE AND STORAGE DURATION ON FISSURING AND MILLING QUALITY OF JASMINE 85 RICE VARIETY
}

\author{
J. O. Akowuah, A. Addo and A. Bart-Plange \\ Department of Agricultural Engineering, KNUST, Kumasi \\ E-mail: akowuahjoe@yahoo.co.uk
}

\begin{abstract}
Moisture and temperature gradients induce the development of fissures during drying of paddy kernels. This affects the milling quality of the paddy and eventually reduces the head rice yield. To this effect, a study was conducted to investigate the influence of drying temperature and storage duration on the cracking behaviour and head rice yield of a local rice variety - Jasmine 85. Freshly harvested paddy samples were thin layer dried in a tunnel dryer with varying drying temperatures $\left(45^{\circ} \mathrm{C}, 50^{\circ} \mathrm{C}, 55^{\circ} \mathrm{C}\right)$ as treatments and direct sun drying as control. The drying experiment was performed under three replications using a completely randomised design. Samples after drying were stored for three months in sealed plastic bags at room temperature $\left(28^{\circ} \mathrm{C}\right) . \mathrm{Per}$ centage fissures and head rice yield at each drying treatment was determined using a grain scope and a Satake grading machine respectively after the storage period. The highest head rice yield (76.3\%) and the least fissure rate (10\%) were attained at the control temperature. This was followed by the $45^{\circ} \mathrm{C}$ and $50^{\circ} \mathrm{C}$ treatments which gave head rice yields of $70.8 \%$, and $69.8 \%$, with fissure rates of $13 \%$ and $16 \%$ respectively. The least head rice yield (64.7\%) and the highest fissure rate of $29 \%$ were observed at $55^{\circ} \mathrm{C}$. There was a significant difference $(p \leq 0.05)$ between head rice yields obtained at $55^{\circ} \mathrm{C}$ and the control experiment. However, there was no significant difference $(p \leq 0.05)$ between head rice yield obtained at $45^{\circ} \mathrm{C}, 50^{\circ} \mathrm{C}$ and the control temperature. It is evident from this study that, Jasmine 85 can be dried by using a mechanical dryer at higher temperatures of $45^{\circ} \mathrm{C}$ and $50^{\circ} \mathrm{C}$ without affecting its milling qualities.
\end{abstract}

Keywords: Jasmine 85, drying temperature, storage duration, fissure formation and head rice yield

\section{INTRODUCTION}

Rice belongs to one of the major economic crops in the world. In Ghana, it is considered as one of the staple cereal crops and is consumed daily in most homes. It is not surprising that, aside the one-third production input by local rice farmers; government spends a whopping 500 million dollars to import the remaining two -thirds to meet the country's requirement annually. Rice is normally harvested at high moisture contents (MCs) of about $20 \%$ to $24 \%$ wet basis (wb). Harvesting at moistures above $25 \%$ is usually unacceptable because of high drying costs and on the average, head rice drops steadily at moistures below 20\% (Cnossen and Siebenmorgen, 2000). Also, Siebenmorgen et al, 
(2007) reported that, optimal harvest moisture content of certain rice cultivars is key to maximising the milling qualities of these varieties. In order to prevent deterioration after harvest, the paddy should be dried down to a safe moisture content of about $12-14 \%$ wb, which is considered adequate for safe storage, milling and further storage as milled rice (Bonazzi et al., 1997; Inprasit and Noomhorm, 2001). It is a common practice for freshly harvested rice to be dried to safe moisture content, typically 12 to $13 \%$ and then stored for some months prior to milling (Hashemi et al., 2008).

During paddy drying, moisture and temperature gradients are created which induces internal cracking of the kernel endosperm and leads to the development of fissures. Schluterman and Siebenmorgen (2007) reported that, hightemperature drying creates moisture content gradients within kernels, which can ultimately lead to fissure formation within the kernels and subsequently reduce milling quality. Fissures drastically reduce the mechanical strength of rice kernels and typically cause kernel breakage during milling, thereby reducing head rice yields (HRYs). Fan et al. (2000) studied the relation between various harvest and drying conditions and its effects on head rice yield reduction of long- and medium-grain rice varieties in the US. Head rice yield is the standard in the rice industry used to measure rice milling quality and is defined as the weight percentage of rough rice that remains as head rice (kernels that are at least three-fourths of the original kernel length) after complete milling.

Investigations on post-drying fissure development of different varieties of paddy under various drying conditions have been undertaken by several researchers. Hashemi et al. (2008) investigated the effects of various drying temperatures, low final moisture, and post-drying duration on fissure formation and head rice yield (HRY) reduction in aromatic short-grain rice. Sarker and Kunze (1996); Jia et al. (2002); Yang et al. (2002) and Zhang et al. (2003) also explained several hypotheses regarding fissur- ing of rice kernels during drying at high temperature and low relative humidity. Cnossen and Siebenmorgen (2000) showed that, certain drying procedures in which kernel temperatures exceeded the kernel's glass transition temperature $\left(\mathrm{T}_{\mathrm{g}}\right)$ lead to fissuring. Sharma and Kunze (1982) reported that most fissures appeared within 48 hours after drying ceased, but some additional fissures appeared up to 120 hours after drying. Other researchers such as Nguyen and Kunze (1984); Sharma and Kunze (1982) observed that fissures did not occur until after drying had ceased.

Kunze and Prasad (1978) reported that kernel fissures created during drying are associated with reabsorption of moisture by low moisture grains. Hashemi et al. (2008) reports that fissuring can occur in the field prior to harvest, harvesting, processing and storage. The rice industry in Ghana is dominated by small scale farmers. Normally, after harvesting and drying rough rice to low final moisture content (FMC) of about $12-14 \%$, the rough rice is stored for long periods before milling depending on market demand for the variety. However, storage of low moisture content grains can reabsorb moisture leading to the development of hydro stresses in the rice kernel, resulting in fissure formation which leads to eventual reduction in head rice yield.

During milling, kernels with fissures tend to break, leading to lower head rice yield recovery. Understanding the effects of rice fissuring is very important to the local rice industry in controlling and optimising drying and milling quality of paddy. The objective of this study therefore was to investigate the influence of drying temperature and storage duration on the cracking behaviour and head rice yield of a local rice variety, Jasmine 85 .

\section{MATERIALS AND METHODS}

Fresh Jasmine 85, an improved local long grain paddy variety with initial moisture content of $22 \%$ wet basis was harvested from a rice farm at Besease, a village in the Ejisu-Juaben Dis- 
trict in the Ashanti Region of Ghana. After harvest, the samples were threshed and cleaned. The initial moisture content was determined in triplicate using an oven method, which comprised drying $15 \mathrm{~g}$ of rough rice for $24 \mathrm{hr}$ in a convection oven at $130^{\circ} \mathrm{C}$ (Jindal and Siebenmorgen, 1987).

Drying experiments were performed in a laboratory model tunnel dryer which included 12 trays $(10 \times 10 \mathrm{~cm})$ with perforated bottoms. The perforated trays made up the drying chamber as described by Zheng (1999) cited in Zheng et al. (2007), where drying air conditions including temperature and relative humidity were controlled by an auto-controller. The drying temperatures were $45^{\circ} \mathrm{C}, 50^{\circ} \mathrm{C}$ and $55^{\circ} \mathrm{C}$ which served as treatments. For statistical control purposes, duplicate samples of rice were sun dried and allowed to dry slowly from the $\mathrm{MC}$ at harvest to approximately $12.5 \% \mathrm{MC}$. Relative humidity of the heated air was at $15 \%$ at an airflow rate of $1.3 \mathrm{~m} / \mathrm{s}$. The paddy samples were spread on each perforated tray containing about $50 \mathrm{~g}$ to form a rice bed of 2 to 3 $\mathrm{cm}$ deep which is within the thickness considered as a thin layer (ASAE, 2001). For each drying air treatment, samples were dried for various durations to produce a range of MC gradients within the kernels. The samples were weighed at time intervals of 5 min until MC of 12 to $14 \%$ was attained. The paddy was taken out and tempered at room temperature to cool down. The samples were then put into sealed plastic bags and stored at room temperature for three months. Drying was repeated in 3 replicates where twelve trays were used for each replication under each drying condition to obtain 36 samples. Combining twelve trays produced enough rice for milling. After the first month of storage, fissure rate and head rice yield were determined. This was repeated after the second and third months of storage.

\section{Fissure rate determination}

The number of fissured kernels was counted in 100 rice kernels which were randomly taken from the control and drying treatment combina- tions and each sample manually dehulled by hand to avoid mechanical damage to the kernels. Fissures were enumerated based on the procedure described by Hashemi et al. (2008), where the kernels were inspected using a grain scope based on light reflection on the grain for the presence of fissures prior to milling. Brown rice kernels were spread on top of the glass cover and inspected with a magnifying glass. Fissured kernels were characterised according to the degree of fissure (heavy and light) and determined in accordance with the Fissure Inspection Standard of the Japanese Society of Agricultural Machinery (JSAM, 1976). Heavy fissures were defined as having internal and/or multiple fissures, and light fissures were defined as fissures occurring on the surface of the kernel. The maximum percentage of fissured kernels was recorded.

\section{Milling tests}

Milling tests were performed by milling $400 \mathrm{~g}$ of the remaining rough rice from each dried sample to determine the head rice yield. A total of 3 HRY determinations were made for each drying treatment and control. The milling procedure consisted of first shelling the $400 \mathrm{~g}$ rough rice using a laboratory huller (Rice Machine THU, Satake Engineering Co., Tokyo, Japan) to obtain brown rice. The brown rice was milled for $35 \mathrm{~s}$ using a laboratory mill (Satake rice polisher). Whole grain rice or head rice was then separated from broken rice using Satake test rice grader (TRC). The HRY was measured based on the percentage of head rice mass remaining from the original $400 \mathrm{~g}$ rough rice sample after complete milling (Cnossen et al., 2000).

\section{RESULTS AND DISCUSSIONS}

Influence of drying temperature and storage duration on head rice yield

Figure 1 shows the change in head rice yield for samples dried at $45^{\circ} \mathrm{C}, 50^{\circ} \mathrm{C}, 55^{\circ} \mathrm{C}$ and the control temperature $\left(34^{\circ} \mathrm{C}\right)$ and placed in sealed bags at room temperature conditions for the indicated post-drying durations. 


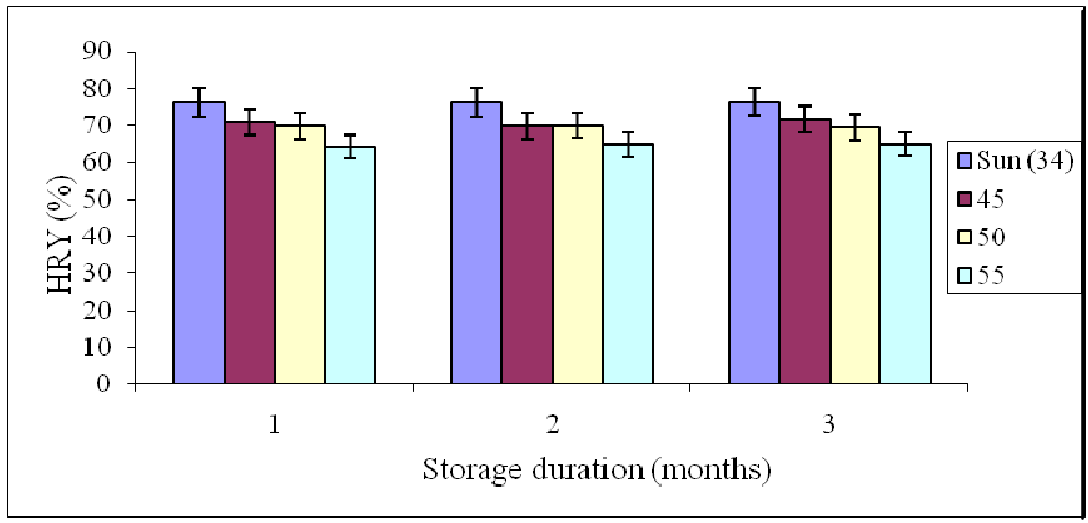

Fig. 1: Effects of drying temperature on head rice yield for specified storage duration

Table 1: Mean head rice yield of Jasmine 85

\begin{tabular}{ll}
\hline $\begin{array}{l}\text { Drying } \\
\text { Temperature }\left({ }^{\mathbf{o}} \mathbf{C}\right)\end{array}$ & $\begin{array}{l}\text { Head rice } \\
\text { yield }(\%)\end{array}$ \\
\hline 45 & $70.8^{\mathrm{a}}$ \\
50 & $69.8^{\mathrm{a}}$ \\
55 & $64.7^{\mathrm{b}}$ \\
Control $(34)$ & $76.3^{\mathrm{a}}$ \\
LSD $(\mathrm{p} \leq 0.05)$ & 0.04 \\
\hline
\end{tabular}

Head rice yield (averaged over three $400 \mathrm{~g}$ subsamples) of samples dried from approximately $22 \%$ to $12.5 \%$ MC using air at the indicated temperatures (constant RH of 15\%) and immediately placed in sealed plastic bags at room temperature for post-drying durations of 1, 2 and 3 months. Means of each column followed by the same letter in the same column are not significantly different at $P \leq 0.05$.

As the drying temperature increased, there was an overall corresponding decrease in HRY which varied with storage duration. From Table 1 , it can be inferred that, the drying temperature had a significant effect $(P \leq 0.05)$ on the HRY for the different post-drying durations.

There was no significant difference $(p \leq 0.05)$ in
HRY reduction for samples dried using the sun $\left(34^{\circ} \mathrm{C}\right)$ and at $45^{\circ} \mathrm{C}$ and $50^{\circ} \mathrm{C}$ drying air temperatures. However, maximum reduction of HRY occurred at $55^{\circ} \mathrm{C}$ drying air temperature for all the post-drying durations. Head rice yield reduction patterns for the various drying air temperature varied with post-drying durations. However, higher drying temperatures produced significant HRY reduction at different post-drying duration. This indicates that the HRY was significantly dependent on drying air temperature. This result is in agreement with the findings of other researchers (Jindal and Siebenmorgen, 1994; Siebenmorgen et al., 2005; Wiset et al., 2001) who reported that the variation in HRY depends on the drying air temperature, fissuring, rigidity, post-drying duration and temperature as well as the grain thickness.

Influence of drying temperature and storage duration on fissure formation

Percentage fissured kernels after the various post-drying durations for each drying air temperature treatment is shown in Figure 2. The average percentage of fissured kernels for the control and treatment samples for the tempera- 
ture treatments $34^{\circ} \mathrm{C}, 45^{\circ} \mathrm{C}, 50^{\circ} \mathrm{C}$ and $55^{\circ} \mathrm{C}$ were $10 \%, 13 \%, 16 \%$ and $29 \%$, respectively. It was observed that increasing drying temperatures resulted in progressive increases in percentage value of fissured kernels. After one month of storage, the percentage of fissured grains increased from $12 \%$ to $31 \%$ with corresponding increase in drying temperature. A similar trend was observed for the other post drying durations as shown in Figure 2. The trend in fissured grains visually correlated with the HRY reductions shown in Figure 1. This trend can be explained by the differences in the rice kernel thermo-physical properties. Perdon et al. (2000) showed that the physical properties of rice kernel changed dramatically as the kernel temperature passed through the glass transition temperature $(\mathrm{Tg})$. Previous research has shown that, the $\mathrm{Tg}$ plays an important role in rice drying and tempering in terms of kernel fissuring potential (Perdon et al., 2000; Cnossen et al., 1999; Cnossen and Siebenmorgen 2000).

According to Slade and Levine (1995), starch, the main constituent of rice, undergoes a glass transition as the kernel temperature increases above the $\mathrm{Tg}$. They reported that, at a temperature and moisture content below the $\mathrm{Tg}$, starch exists as a 'glassy' material, with low expansion coefficients, specific volume, and diffusiv- ity. As the kernel temperature increases above the Tg, the starch transitions from a 'glassy' into a 'rubbery' state with higher expansion coefficients, specific volume, and diffusivity. As shown in Figure 3, Perdon (1999) cited in Cnossen and Siebenmorgen (2000) illustrates the $\mathrm{Tg}$ relationship of a rice variety indicating the glassy region and the rubbery region.

The changes in volumetric expansion coefficient and specific volume when transitioning from the glassy to the rubbery region according to Perdon et al. (2000) could have an effect on kernel fissuring, while the changes in diffusivity would greatly affect drying and tempering rates.

Siebenmorgen and Schluterman (2007) also reported that, extended drying creates intrakernel moisture content gradients, causing the kernel surface to dry to low moisture contents, thereby causing sufficient portions of the kernel periphery to transition into the glassy state while the center remains in the rubbery state. The dramatic differences in kernel properties between these states, particularly expansion rates leads to fissure formation and resultant HRY reductions. The results also support the $\mathrm{Tg}$ hypothesis proposed by Cnossen and Siebenmorgen (2000).

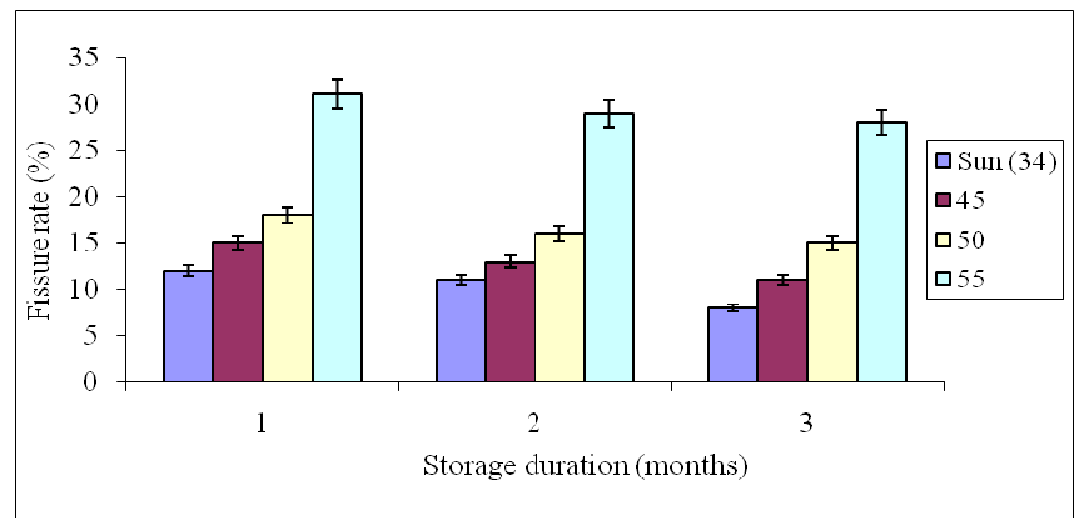

Fig. 2: Average percentage fissured grains observed over three month's storage duration at the indicated drying temperatures 


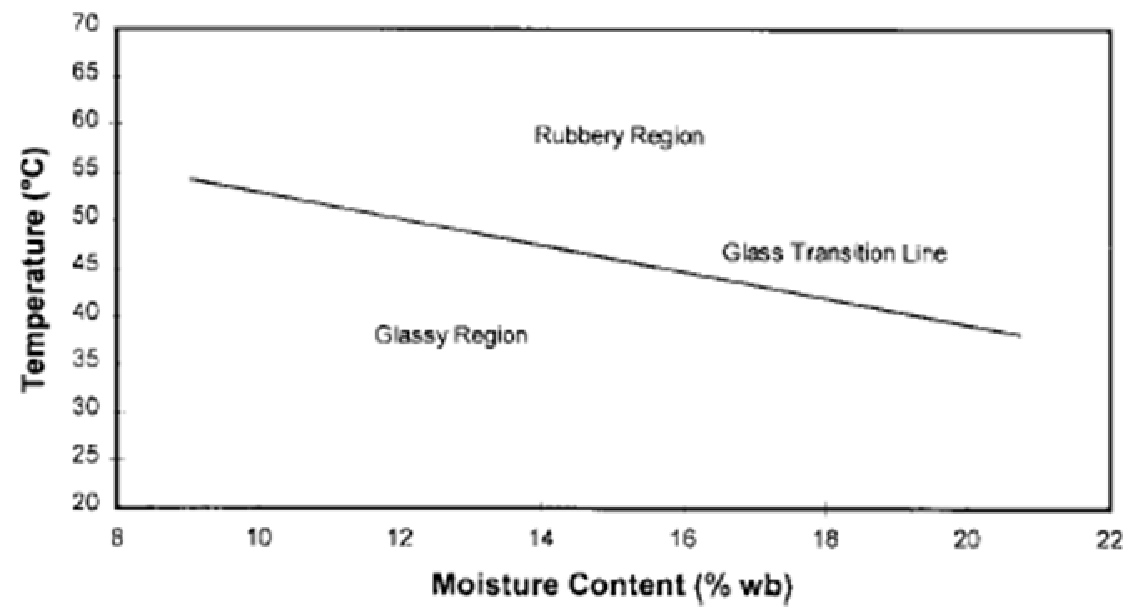

Fig. 3: Glass transition relationship of brown rice indicating the glassy and the rubbery region

\section{CONCLUSIONS}

Hot air drying experiments using a laboratory scale tunnel dryer and open sun drying were conducted to investigate the effect of drying air temperatures $\left(45^{\circ} \mathrm{C}, 50^{\circ} \mathrm{C}, 55^{\circ} \mathrm{C}\right.$ and open sun drying) and post-drying duration on fissuring and head rice yield of a local long-grain rice variety (Jasmine 85). Milling quality of rice was found to be affected by drying temperature. Higher temperatures resulted in lower HRY and increased fissure formation. Induced fissures and head rice yield had a direct correlation with the drying air temperature during drying and milling of the paddy. For post-drying duration (storage duration) of 1, 2 and 3 months, paddy dried at $55^{\circ} \mathrm{C}$ gave the highest fissure rate and lowest head rice yield while paddy dried at the sun's temperature $\left(34^{\circ} \mathrm{C}\right)$ resulted in the lowest induced fissure rate and highest head rice yield. However, there was no significant difference $(P$ $\leq 0.05$ ) in head rice yield of paddy dried at air temperatures of $45^{\circ} \mathrm{C}, 50^{\circ} \mathrm{C}$ and the control temperature $\left(34^{\circ} \mathrm{C}\right)$ for all the post-drying durations. This presumes that, paddy rice can be dried at temperatures of $45^{\circ} \mathrm{C}$ and $50^{\circ} \mathrm{C}$ using a mechanical dryer when the harvest and raining season coincides.

\section{REFERENCES}

ASAE (2001). ASAE Standards, Standard S448.1. Thin-layer drying of grains and crops. ASAE. St. Joseph, MI.

Bonazzi, C., Peuty M. and Themelin, A. (1997). Influence of drying conditions on the processing quality of rough rice. Drying Tech. 15(3/4): 1141-1157.

Cnossen, A. G. and Siebenmorgen, T. J. (2000). The glass transition temperature concept in rice drying and tempering: Effect on milling quality. Transactions of the ASAE 43: 16611667.

Cnossen, A. G. and Siebenmorgen, T. J. (1999). Effect of tempering temperature and duration on rice milling quality. ASAE Paper No. 016117. St. Joseph, Mich.: ASAE.

Fan, J., Siebenmorgen, T. J. and Yang, W. (2000). A study of head rice yield reduction of long- and medium-grain rice varieties in relation to various harvest and drying conditions. Trans. ASAE 43(6):1709-1714.

Hashemi, J., Haque, A., Shimizu, N. and Ki- 
mura, T. (2008). Influence of Post drying Duration on the Head Rice Yield of Aromatic Rice. Agricultural Engineering International: the CIGR Ejournal. Manuscript FP 07034. Vol. X.

Inprasit, C. and Noomhorm, A. (2001). Effect of drying air temperature and grain temperature of different types of dryer and operation on rice quality. Drying Tech. 19(2): 389-404.

JSAM (1976). Proposal on criteria of measuring method for rice fissure. Journal of Japanese Society of Agricultural Machinery 38 (2): 253-254.

Jia, C. C., Yang, W., Siebenmorgen, T. J., Bautista, R. C. and Cnossen, A. G. (2002). A study of rice fissuring by finite-element simulation of internal stresses combined with high-speed microcopy imaging of fissure appearance. Transactions of the ASAE 45: 741-749.

Jindal, V. K. and Siebenmorgen, T. J. (1994). Simulation of low temperature rough rice drying and rewetting in shallow beds. Transactions of the ASAE 37: 863-871.

Jindal, V. K. and Siebenmorgen, T. J. (1987). Effects of oven drying temperature and drying time on rough rice moisture content determination. Transactions of the ASAE 30 (4):1185-1192.

Kunze, O. R. and Prasad, S. (1978). Grain fissuring potentials in harvesting and drying of rice. Transactions of the ASAE 21(2):361366.

Nguyen, C. N. and Kunze, O. R. (1984). Fissures related to post-drying treatments in rough rice. Cereal Chem. 61(1):63-68.

Perdon, A. A., Siebenmorgen, T. J. and Mauromoustakos, A. (2000). Glassy state transition and rice drying: Development of a brown rice state diagram. Cereal Chem. 77(6): 708-713
Slade, L. and Levine, H. (1995). Glass transitions and water-Food structure interactions. Adv. Food Nutrit. Res. 38: 103-269.

Siebenmorgen, T. J., Qin, G. and Jia, C. (2005). Influence of drying on rice fissure formation rates and mechanical strength distributions. Transactions of the ASAE 48: 1835-1841.

Siebenmorgen, T. J., Bautista, R. C. and Counce, P. A. (2007). Optimal harvest moisture content for maximizing milling quality of long- and medium-grain rice cultivars. Applied Engineering in Agriculture. 23(4): 517-527.

Schluterman, D. A. and Siebenmorgen, T. J. (2007). Relating rough rice moisture content reduction and tempering duration to head rice yield reduction. Transactions of the ASAE 50 (1):137-142.

Sharma, A. D. and Kunze, O. R. (1982). Postdrying fissure development in rough rice. Trans. ASAE 25(2): 465-468, 474.

Sarker, N. N. and Kunze, O. R. (1996). Transient moisture gradients in rough rice mapped with finite element model and related to fissure after heated air drying. Transactions of the ASAE 39: 625-635.

Wiset, L., Srzednicki, G., Driscoll, R., Nimmuntavin, C. and Siwapornrak, P. (2001). Effects of high temperature drying on rice quality. The CIGR Journal of Scientific Research and Development. 01003 (3): 1-10.

Yang, W., Jia, C., Siebenmorgen, T. J., Howell, T. A. and Cnossen, A. G. (2002). Intra-kernel moisture responses of rice to drying and tempering treatments by finite-element simulation. Transactions of the ASAE 45: 10371044.

Zhang, Q., Yang, W., Howard, L. and Earp, C. F. (2003). Tracing fissure information by scanning electron microscopy characteriza- 
tion of naturally fissured surfaces of rice kernels. Transactions of the ASAE 46: 15831588 .

Zheng, X. and Lan, Y. (2007). Effects of drying temperature and moisture content on rice taste quality. Agricultural Engineering International: the CIGR Ejournal. Manuscript FP07 023, Vol. IX. 